\title{
Frustration-driven magnetic fluctuations as the origin of the low-temperature skyrmion phase in $\mathrm{Co}_{7} \mathrm{Zn}_{7} \mathrm{Mn}_{6}$
}

V. Ukleev $\mathbb{D}^{1 凶}$, K. Karube ${ }^{2}$, P. M. Derlet ${ }^{3}$, C. N. Wang ${ }^{4}$, H. Luetkens $\mathbb{D}^{4}$, D. Morikawa ${ }^{2}$, A. Kikkawa ${ }^{2}$, L. Mangin-Thro $\mathbb{D}^{5}$, A. R. Wildes ${ }^{5}$, Y. Yamasaki ${ }^{6,7}$, Y. Yokoyama ${ }^{6}$, L. Yu ${ }^{1,8,9}$, C. Piamonteze ${ }^{10}$, N. Jaouen $\mathbb{D}^{11}{ }^{11}$, Y. Tokunaga ${ }^{12}$, H. M. Rønnow $\mathbb{D}^{13}$, T. Arima ${ }^{2,12}$, Y. Tokura $\mathbb{D}^{2,14,15}$, Y. Taguchi $\mathbb{D}^{2}$ and J. S. White $\mathbb{D D}^{1 凶}$

In chiral cubic helimagnets, phases of magnetic skyrmions-topologically protected spin whirls-are stabilized by thermal fluctuations over a narrow region directly below the magnetic ordering temperature $T_{\mathrm{c}}$. Due to often being touted for use in applications, there is a high demand to identify new ways to stabilize equilibrium skyrmion phases far below $T_{\mathrm{c}}$ where they may display an enhanced robustness against external perturbation due to a larger magnetic order parameter. Here, from quantum beam experiments on the chiral magnet $\mathrm{Co}_{7} \mathrm{Zn}_{7} \mathrm{Mn}_{6}$, we unveil a direct correlation between the stability of its second skyrmion phasestable far from $T_{c}$, and a concomitant enhancement of an underlying magnetic fluctuation rate that is driven by geometric magnetic frustration. The influences of other leading skyrmion stability mechanisms, such as those derived from thermal fluctuations and low $T$ cubic anisotropies, are shown to be weak in this system. We therefore advance the existence of a fundamental mechanism for stabilizing topological skyrmions in $\mathrm{Co}_{7} \mathrm{Zn}_{7} \mathrm{Mn}_{6}$ chiral magnet that draws upon magnetic frustration as the key ingredient.

npj Quantum Materials (2021)6:40; https://doi.org/10.1038/s41535-021-00342-5

\section{INTRODUCTION}

In non-centrosymmetric magnets, the interplay between ferromagnetic (FM) exchange, Dzyaloshinskii-Moriya interactions, and magnetic anisotropy, results in rich phase diagrams containing helical, conical and skyrmion lattice phases ${ }^{1-3}$. Skyrmion phases are the most intriguing, since each skyrmion displays a topologically nontrivial spin texture that is easily manipulated by external stimuli ${ }^{4-6}$. This makes skyrmions promising for diverse applications such as high-density spintronics and neuromorphic computing $2,7,8$.

Chiral cubic magnets such as $\mathrm{MnSi}^{9}, \mathrm{FeGe}^{10}, \mathrm{Cu}_{2} \mathrm{OSeO}_{3}{ }^{11}$, and Co-Zn-Mn compounds ${ }^{12-16}$ are the archetypal skyrmion host materials. All of these systems display commonalities amongst their phase diagrams, including the onset of helimagnetic order on zero-field cooling (ZFC) below the critical temperature $T_{\mathrm{c}}$ and a skyrmion lattice (SkL) phase, known as the A-phase, stabilized in a finite magnetic field $(H)$ and over a narrow $T$-range directly below $T_{\mathrm{c}}^{9,11-16}$. The proximity of the A-phase to $T_{\mathrm{c}}$ supports theoretical suggestions that thermal fluctuations are a key ingredient for Aphase stability, this being akin to an order-by-disorder mechanism $^{9,17-21}$. It is further proposed that quantum fluctuations can stabilize skyrmions as well ${ }^{22}$.

The Co- $\mathrm{Zn}-\mathrm{Mn}$ series $\left(\mathrm{Co}_{0.5} \mathrm{Zn}_{0.5}\right)_{20-x} \mathrm{Mn}_{x}, x=0-20$, all crystallize in the chiral cubic $\beta$-Mn-type structure (space group $P 4_{1} 32 /$ $P_{4} 32$ ) with 20 atoms per unit cell distributed across two Wyckoff sites, the $8 c$ and $12 d$ sites [Fig. 1a]. Previous work reveals that Co tends to occupy the $8 c$ site, while $\mathrm{Zn}$ and Mn tend to occupy the $12 d$ site ${ }^{12,15,23,24}$. The magnetic properties vary strongly with $x$; the $\mathrm{Mn}$-free end member $\mathrm{Co}_{10} \mathrm{Zn}_{10}(x=0)$ displays a high- $T$ helical ordering of Co spins below $T_{\mathrm{c}} \sim 415 \mathrm{~K}^{16}$. As $\mathrm{Mn}$ substitution proceeds, $T_{\mathrm{c}}$ falls quickly to $\sim 300 \mathrm{~K}$ in $\mathrm{Co}_{8} \mathrm{Zn}_{8} \mathrm{Mn}_{4}$, and $\sim 160 \mathrm{~K}$ in $\mathrm{Co}_{7} \mathrm{Zn}_{7} \mathrm{Mn}_{6}$. Nonetheless all systems from $0 \leq x \leq 6$ display an $\mathrm{A}-$ phase close to $T_{c}$ that is stable either above, at, or below room $T$, depending on $x^{12,15,16,24}$. The other end member $\operatorname{Mn}_{20}(x=20), \beta$ $\mathrm{Mn}$ itself, is an elemental geometrically frustrated spin liquid due to antiferromagnetic (AFM) interactions between Mn moments on the hyper-kagomé coordinated $12 d$ site $^{25-27}$. For $3 \leq x \leq 7$ a transition to a spin glass takes place below a temperature $T_{\mathrm{g}}<$ $T_{c}$. This regime is indicative for persistent magnetic frustration effects inherited from $\beta-\mathrm{Mn}$, that are found to co-exist with any pre-existing helical spin correlations ${ }^{12,13,15,16,23,24}$.

Here, we focus on $\mathrm{Co}_{7} \mathrm{Zn}_{7} \mathrm{Mn}_{6}(x=6)$, a mixed composition system displaying the unusual effects of intertwined chiral and frustrated magnetism. Remarkably this system hosts two equilibrium skyrmion phases ${ }^{15}$, these being the A-phase, and an additional low-temperature skyrmion (LTSk) phase that was unambiguously identified by small-angle neutron scattering (SANS) and Lorentz transmission electron microscopy (LTEM) and called a 'disordered' skyrmion phase in ref. ${ }^{15}$. As summarized in (Fig. 1b), the LTSk phase is stable in finite $H$ and for temperatures just above $T_{\mathrm{g}} \sim 35 \mathrm{~K}$, this being far from the Aphase stable near $T_{\mathrm{c}}$. With thermal fluctuations suppressed far from $T_{\mathrm{c}}$, multiple skyrmion stability mechanisms can be supposed

\footnotetext{
'Laboratory for Neutron Scattering and Imaging (LNS), Paul Scherrer Institute (PSI), Villigen, Switzerland. ${ }^{2}$ RIKEN Center for Emergent Matter Science (CEMS), Wako, Japan. ${ }^{3}$ Condensed Matter Theory Group, Paul Scherrer Institute (PSI), Villigen, Switzerland. ${ }^{4}$ Laboratory for Muon Spin Spectroscopy (LMU), Paul Scherrer Institute (PSI), Villigen, Switzerland. Institut Laue-Langevin, Grenoble, France. ${ }^{6}$ Research and Services Division of Materials Data and Integrated System (MaDIS), National Institute for Materials Science (NIMS), Tsukuba, Japan. ${ }^{7}$ PRESTO, Japan Science and Technology Agency (JST), Kawaguchi, Japan. ${ }^{8}$ Laboratory for Ultrafast Microscopy and Electron Scattering (LUMES), Institute of Physics, École Polytechnique Fédérale de Lausanne (EPFL), Lausanne, Switzerland. ${ }^{9}$ Laboratory of Nanoscale Magnetic Materials and Magnonics (LMGN), Institute of Materials, École Polytechnique Fédérale de Lausanne (EPFL), Lausanne, Switzerland. ${ }^{10}$ Swiss Light Source, Paul Scherrer Institute (PSI), Villigen, Switzerland. ${ }^{11}$ Synchrotron SOLEIL, Gif-surYvette Cedex, France. ${ }^{12}$ Department of Advanced Materials Science, University of Tokyo, Kashiwa, Japan. ${ }^{13}$ Laboratory for Quantum Magnetism (LQM), Institute of Physics, École Polytechnique Fédérale de Lausanne (EPFL), Lausanne, Switzerland. ${ }^{14}$ Department of Applied Physics, University of Tokyo, Tokyo, Japan. ${ }^{15}$ Tokyo College, University of Tokyo, Tokyo, Japan. ${ }^{\bowtie}$ email: victor.ukleev@psi.ch; jonathan.white@psi.ch
} 
(a)

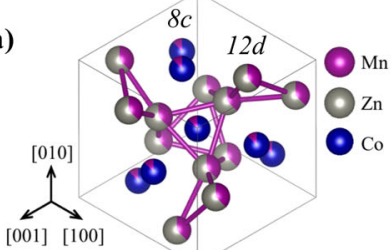

(b)

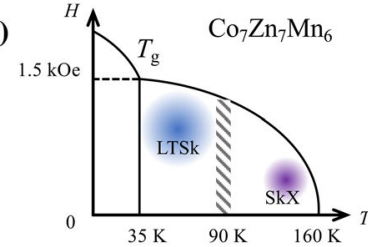

(c)

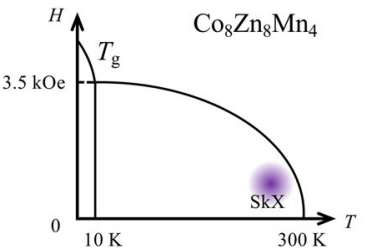

(d)

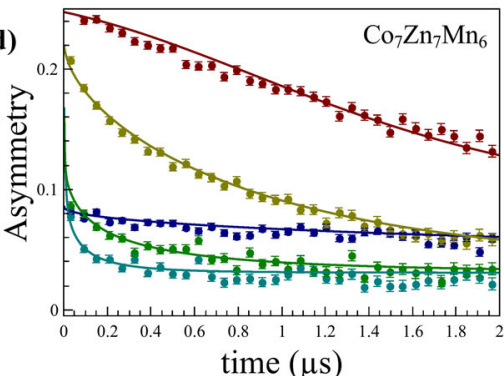

(e)

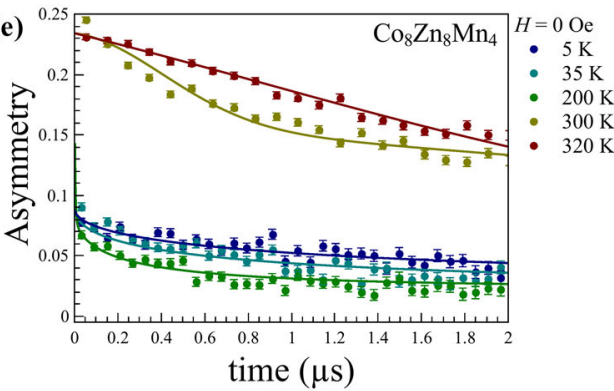

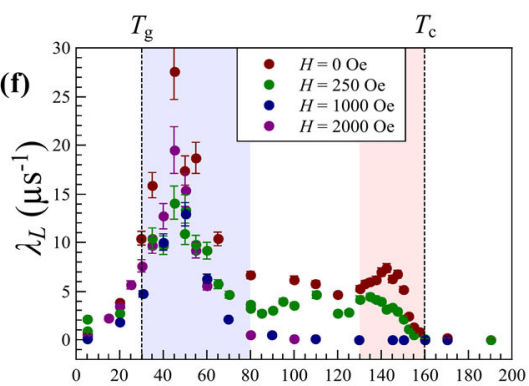

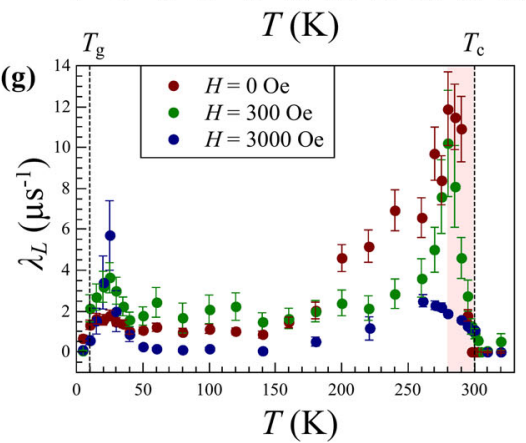

Fig. 1 Crystal structure, magnetic phase diagrams and muon spectroscopy of CoZnMn alloys. a $\beta$-Mn-type structure (space group: $P 4,32$ ), comprising $8 c$ and $12 d$ atomic sites, as viewed along the [111] axis. The bonds between $\mathrm{Mn}$ atoms at $12 d$ sites are shown. Schematic $T-H$ magnetic phase diagram in (b) $\mathrm{Co}_{7} \mathrm{Zn}_{7} \mathrm{Mn}_{6}$ and (c) $\mathrm{Co}_{8} \mathrm{Zn}_{8} \mathrm{Mn}_{4}$ obtained from SANS and susceptibility measurements ${ }^{13,15}$. Measured (symbols) and fitted (solid lines) $\mu \mathrm{SR}$ spectra at selected temperatures for $\mathrm{Co}_{7} \mathrm{Zn}_{7} \mathrm{Mn}_{6}(\mathbf{d})$ and $\mathrm{Co}_{8} \mathrm{Zn}_{8} \mathrm{Mn}_{4}(\mathbf{e})$. Corresponding temperature dependencies of relaxation rates $\lambda(T)$ obtained from the fits are shown in (f) and (g) for $\mathrm{Co}_{7} Z n_{7} \mathrm{Mn}_{6}$ and $\mathrm{Co}_{8} Z \mathrm{n}_{8} \mathrm{Mn}_{4}$, respectively. The temperature ranges for SkL and LTSk phases are indicated by purple and blue background colours, respectively. In (d, e), error bars indicate the statistical error. In (f, g), error bars are obtained from the fitted model described in the main text.

to exist in this single chiral magnet. In the following, we apply an arsenal of quantum beam probes (muons, neutrons, X-rays and electrons), to elucidate the microscopic magnetism and dynamics across the phase diagram of $\mathrm{Co}_{7} \mathrm{Zn}_{7} \mathrm{Mn}_{6}$, from atomic-tonanometric length-scales, and $\mu$ s-ps time scales. We find that the LTSk phase is stable over a thermal range characterized by an enhanced magnetic fluctuation rate on the $\mu$ s time scale, the origin of which is connected to the geometric magnetic frustration of $\mathrm{Mn}$ moments on the $12 d$ site. Our experiments thus uncover an alternative mechanism for stabilizing topological skyrmions in chiral magnets that draws upon magnetic frustration as the key ingredient.

\section{RESULTS AND DISCUSSION}

\section{Experimental observation of magnetic fluctuations by $\boldsymbol{\mu S R}$}

Muon spin relaxation ( $\mu \mathrm{SR}$ ) experiments were performed to determine the $H$ and $T$-dependent characteristic magnetic fluctuation rates in $\mathrm{Co}_{7} \mathrm{Zn}_{7} \mathrm{Mn}_{6}$. These experiments are sensitive to time scales ranging from $10^{-12}$ to $10^{-4} \mathrm{~s}$ depending on the size of the magnetic field at the muon site. Figure $1 \mathrm{~b}$ shows $\mu \mathrm{SR}$ spectra recorded from bulk single-crystal $\mathrm{Co}_{7} \mathrm{Zn}_{7} \mathrm{Mn}_{6}$ across its entire phase diagram using the general purpose surface (GPS) instrument at the Paul Scherrer Institute. Further experimental details are given in the "Methods" section. For comparative purposes, further data were obtained similarly from the sister compound $\mathrm{Co}_{8} \mathrm{Zn}_{8} \mathrm{Mn}_{4}(x=4)$ with $T_{\mathrm{g}} \sim 10 \mathrm{~K}$, and in which no LTSk phase is known to exist (Fig. 1c).

Figure $1 \mathrm{~d}$, e shows selected time spectra measured on warming after an initial ZFC, and in zero magnetic field (ZF). For $T>T_{\mathrm{c}}$, the $T$ dependent muon depolarization is dominated by fast paramagnetic spin fluctuations. As done previously for $\beta-\mathrm{Mn}^{28}$, we fit these high $T$ data with a muon depolarization function $A(t)$ described by a product of a $T$-independent Gaussian Kubo-Toyabe relaxation describing static nuclear moments, and another exponential term $\exp \left(-\lambda_{\mathrm{L}} t\right)$ that accounts for fluctuating electronic moments: $A(t) / A_{0}=\left[1 / 3+2 / 3\left(1-\sigma^{2} t^{2}\right) \cdot \exp \left(-\sigma^{2} t^{2} / 2\right)\right] \cdot \exp \left(-\lambda_{\mathrm{L}} t\right)$. Here, $A_{0}$ is the total asymmetry, $t$ is time, $\sigma=\gamma_{\mu} \sqrt{\left\langle\Delta B_{\text {loc }}^{2}\right\rangle}=0.359 \pm 0.025$ $\mu \mathrm{s}^{-1}$ is the width of the local nuclear spin field distribution $\left\langle\Delta B_{\text {loc }}\right\rangle_{,} \gamma_{\mu}$ is the muon gyromagnetic ratio, and $\lambda_{L}$ is the muon relaxation rate. In the fast fluctuating limit, the correlation time of the fluctuating moment is proportional to $\lambda_{\mathrm{L}}$.

Below $T_{\mathrm{c}}$, the $t$-dependent muon depolarization in both systems reveals a slowing down of spin dynamics, particularly on cooling towards lower $T$. This is indicated by the initial fast depolarization of the so-called transverse part of the spectrum, followed by a slow depolarization of the longitudinal part. This separation into transverse and longitudinal relaxations far below $T_{\mathrm{c}}$ indicates clearly that each system is in a slow fluctuation regime where the magnetic fluctuation rate is much smaller than the characteristic Larmor frequency of the muon spin precession ${ }^{29}$, and can be calculated as $v=3 \lambda_{L} / 2$, where $\lambda_{L}$ is the muon longitudinal relaxation rate. Within this slow fluctuation regime, the muon depolarization function that well-describes the data is given by $A(t) / A_{0}=f_{\mathrm{T}} \cdot \exp \left[-\left(\lambda_{\mathrm{T}} t\right)\right]+f_{\mathrm{L}} \cdot \exp \left(-\lambda_{\mathrm{L}} t\right)^{\beta_{L}}$. Here, $f_{\mathrm{L}}$ and $f_{\mathrm{T}}$ are respectively the longitudinal and transverse fractions of the asymmetry, $\lambda_{\mathrm{L}}$ and $\lambda_{\mathrm{T}}$ are the muon relaxation rates for each component, respectively, and $\beta_{L}$ is an exponent that indicates the distribution of depolarization rates ${ }^{30}$. Convergent fits with $\chi^{2} \approx 1$ were achieved for fitted $f_{\mathrm{T}}=1-f_{\mathrm{L}}$ and fixed $\beta_{\mathrm{L}}=0.5$. Notably, the fitted values of $f_{\mathrm{L}}=0.31 \pm 0.07$ are close to the value of $1 / 3$ expected due to magnetic order in a polycrystalline sample. In the present case for the single crystal, $f_{\mathrm{L}} \sim 1 / 3$ due to the onset of the complex multi-domain helicoidal spin structures known to exist below $T_{\mathrm{c}}{ }^{15}$

From our fitting of all data, we obtained the $T$-dependence of the magnetic relaxation rates $\lambda_{\mathrm{L}}$ and $\lambda_{\mathrm{T}}$, which inform about the spatial and temporal internal field distributions. In both compounds, fitting of the data near $T_{\mathrm{c}}$ provides a clear indication of 
the magnetic ordering transition [see Supplementary note 1], with the onset of the fast relaxation $\lambda_{T}(T)$ [see Supplementary fig. 1] coinciding with the onset of long-range helical order below $T_{\mathrm{c}}^{13,15}$. Below $T_{\mathrm{c}}$, the transverse component of the spectrum depolarizes so quickly that it becomes barely observable in the muon time window. In contrast, the fitted longitudinal component $\lambda_{L}(T)$ is more revealing; Fig. 1f, g each show $\lambda_{\mathrm{L}}(T)$ to display two maxima in both ZF and a low longitudinal magnetic field (LF); one just below $T_{c}$ and one just above $T_{\mathrm{g}}$. Each maximum denotes a $T$ range characterized by an enhanced relaxation rate. The high- $T$ maximum is suppressed under increased LF as expected ${ }^{31}$, while the low $T$ peak shows only a weak LF-dependence.

While a regime of enhanced magnetic relaxation underlying skyrmion phases near $T_{\mathrm{c}}$ has been commonly observed by $\mu \mathrm{SR}^{32-36}$, the existence of a second maximum just above $T_{\mathrm{g}}$ is hitherto unique to $\mathrm{Co}_{7} \mathrm{Zn}_{7} \mathrm{Mn}_{6}$ and $\mathrm{Co}_{8} \mathrm{Zn}_{8} \mathrm{Mn}_{4}$ amongst skyrmion hosts. Importantly for $\mathrm{Co}_{7} \mathrm{Zn}_{7} \mathrm{Mn}_{6}$, the low $T$ peak in $\lambda_{\mathrm{L}}$ coincides with the thermal range of the LTSk phase, thus connecting the phase stability to the enhancemed magnetic fluctuation rate. In addition, the maximal $v_{\max }=3 \lambda_{\mathrm{Lmax}} / 2 \approx 40 \mathrm{MHz}$ is considerably higher than that for $\mathrm{Co}_{8} \mathrm{Zn}_{8} \mathrm{Mn}_{4} \quad(\approx 9 \mathrm{MHz})$ wherein no LTSk phase exists, implying LTSk phase stability to depend further on the magnitude of the low- $T$ fluctuation rate.

The $T$-dependent peaks in $\lambda_{\mathrm{L}}(T)$ reported in Fig. 1f, g display clear resemblance with other magnetically frustrated systems such as re-entrant spin glasses ${ }^{37-43}$ and moreover other $\beta-\mathrm{Mn}$ type compounds ${ }^{28,38}$. In binary $\beta-\mathrm{Mn}_{0.81}-\mathrm{Ru}_{0.19}$ for example, the analogous coexistence of static order and slow magnetic dynamics is substantiated by $T$-dependent peaks in muon relaxation rate observed near both $T_{\mathrm{g}}$ and also the Néel $T^{44}$, and further by quasi-elastic neutron scattering ${ }^{45}$. This comparison implicates the physics of magnetic frustration in binary $\beta$ $\mathrm{Mn}$ compounds to be also present in isostructural $\mathrm{Co}_{7} \mathrm{Zn}_{7} \mathrm{Mn}_{6}$ and $\mathrm{Co}_{8} \mathrm{Zn}_{8} \mathrm{Mn}_{4}$. Moreover, since convincing models for the magnetism in frustrated $\beta-\mathrm{Mn}$ systems are derived for nonmagnetic $8 c$ sites $^{27,46-48}$, it becomes plausible that the peak in $\lambda_{\mathrm{L}}(T)$ we observe near $T_{\mathrm{g}}$ in $\mathrm{Co}_{7} \mathrm{Zn}_{7} \mathrm{Mn}_{6}$ and $\mathrm{Co}_{8} \mathrm{Zn}_{8} \mathrm{Mn}_{4}$ are similarly due to fluctuations of frustrated $\mathrm{Mn}$ moments on the $12 d$ site. The deduced importance of the Mn fluctuations is substantiated by comparing the $\mu \mathrm{SR}$ obtained from the two compounds. The peak in $\lambda_{\mathrm{L}}(T)$ just above $T_{\mathrm{g}}$ is smaller in $\mathrm{Co}_{8} \mathrm{Zn}_{8} \mathrm{Mn}_{4}$ which has a reduced $\mathrm{Mn}$ content compared with $\mathrm{Co}_{7} \mathrm{Zn}_{7} \mathrm{Mn}_{6}$, and thus clear signs of magnetic frustration relief in combination with the higher $T_{\mathrm{c}}$ and lower $T_{\mathrm{g}}$.

\section{Short-range magnetic correlations observed by diffuse neutron scattering}

To obtain further evidence for the frustrated magnetism of $\mathrm{Mn}$ at the $12 d$ site in $\mathrm{Co}_{7} \mathrm{Zn}_{7} \mathrm{Mn}_{6}$, we performed magnetic diffuse neutron scattering (MDNS) measurements of short-range spin correlations. The experiment integrated the spin fluctuations over energy scales of the order of $\mathrm{THz}$, and thus over time scales longer than a few picoseconds. Here, microsecond spin fluctuations observed in the $\mu \mathrm{SR}$ are static within the timeframe of the present MDNS experiments, and thus they may contribute to the observed signal. Low $T$ MDNS measurements were done on $\mathrm{Co}_{7} \mathrm{Zn}_{7} \mathrm{Mn}_{6}$ powder using the D7 spectrometer at the ILL, France ${ }^{49}$, and using an $x y z$ polarization analysis to isolate the magnetic scattering component $^{50}$ - see the "Methods" section for further experimental details. Figure 2 shows the magnetic diffuse scattering profile obtained at $2 \mathrm{~K}$ and in ZF. The profile contains magnetic Bragg peaks shown in light grey, arising mainly from the longrange order of Co moments at the $8 c$ site $^{23}$. The peak of MDNS shown in blue is a hallmark of short-range spin correlations often found in frustrated magnets ${ }^{51}$. Notably, the MDNS peaks near to $Q=1.6 \AA^{-1}$ similarly as for other $\beta$-Mn systems with similar cubic lattice constants ${ }^{27,50}$. Higher $T$ profiles shown in Supplementary

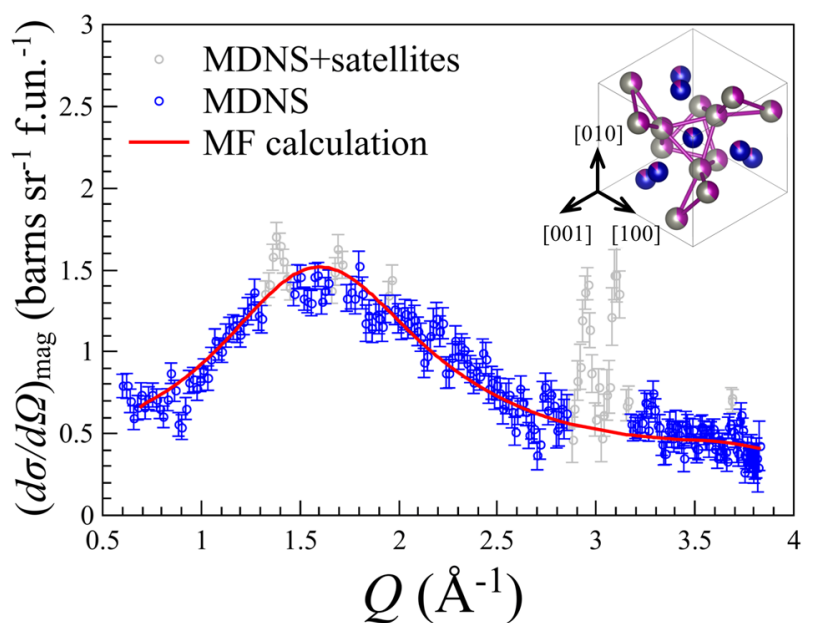

Fig. 2 Neutron scattering from $\mathrm{Co}_{7} \mathrm{Zn}_{7} \mathrm{Mn}_{6}$ and mean-field model. Magnetic scattering measured at $\mathrm{D} 7$ from $\mathrm{Co}_{7} \mathrm{Zn}_{7} \mathrm{Mn}_{6}$ powder at $T$ $=2 \mathrm{~K}$. The entire profile is shown by blue and light grey symbols. The latter correspond to magnetic Bragg peaks, and are omitted for the MF $J_{n n}$ model fitting described in the text (red line). Error bars indicate the statistical error. Inset: Sketch showing the $J_{\mathrm{nn}}$ interaction (purple bonds) between nearest-neighbours on the $12 d$ site.

Fig. 2 reveal the volume of MDNS falls monotonically with increasing $T$, and that it persists for high $T>T_{\mathrm{c}}$.

To determine the origin of the MDNS we fit the blue data portion of the profile in Fig. 2 by a mean-field (MF) model that calculates the paramagnetic neutron scattering for AFM interacting $\mathrm{Mn}$ moments at the $12 d$ sites. Further details of the calculation and our assumptions are given in the "Methods". Optimal values of the exchange constants at short $\mathrm{Mn}-\mathrm{Mn}$ distances were obtained by direct fitting to the data. The red line in Fig. 2 shows a fit to a minimal model that considers a single nearest neighbour (inset Fig. 2) AFM exchange constant $J_{\mathrm{nn}} \approx-0.54 \mathrm{meV}$ with a noninteracting $T$-dependent spin susceptibility taken into account. From extensive fitting, we find that a single parameter nearest neighbour AFM exchange constant is sufficient to optimally reproduce the experimental data with only $J_{\mathrm{nn}} \approx-0.54 \mathrm{meV}$. The inclusion of more distant exchanges does not result in a significantly improved fit, though their importance can be tested against future single-crystal measurements ${ }^{27}$ (see Supplementary note 2). Such a nearest neighbour AFM exchange interaction also gives a MF spin-pair correlation function $\langle S(0) \cdot S(r)\rangle$ in reasonable agreement with that obtained directly from the data via reverse Monte Carlo (RMC) refinement (see Supplementary note 3 ).

Overall, the analysis suggests nearest neighbour AFM interactions between $\mathrm{Mn}$ moments on the $12 d$ site as a plausible origin for the observed MDNS. It follows that due to the hyper-kagomé geometry of the $12 d$ site, geometrical frustration is implicated as the crucial ingredient that suppresses the long-range order of $\mathrm{Mn}$ moments. Instead, from $\mu$ SR we find these Mn moments fluctuate on $\mu$ s time scales over an extended $T$-range down to $T_{\mathrm{g}}$, this being consistent with their appearance as static short-range spin correlations in DNS which is sensitive to faster time scales.

\section{Magnetic element-selective resonant X-ray scattering}

To further substantiate our deductions on the existence of geometric frustration-induced fluctuations of $\mathrm{Mn}$ moments underlying LTSk phase stability in $\mathrm{Co}_{7} \mathrm{Zn}_{7} \mathrm{Mn}_{6}$, we next consider magnetic element-selective resonant $X$-ray scattering experiments performed at the $L_{2,3}$ edges of $C_{0}$ and $M n$ using the XTreme beamline at the SLS facility, PSI, Switzerland ${ }^{52}$, and SEXTANTS beamline, Soleil Synchrotron, France ${ }^{53}$. Further experiment details are given in the Methods. 


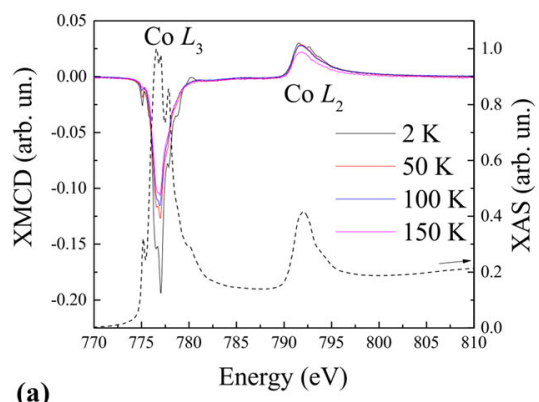

(a)

(d) REXS experiment

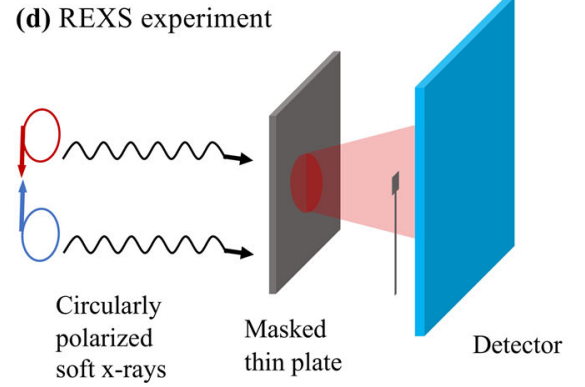

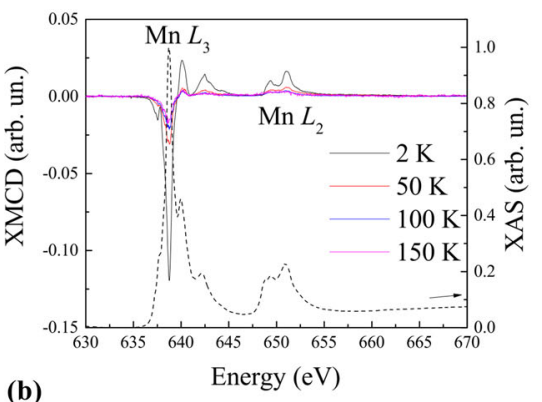

(b)

(e)

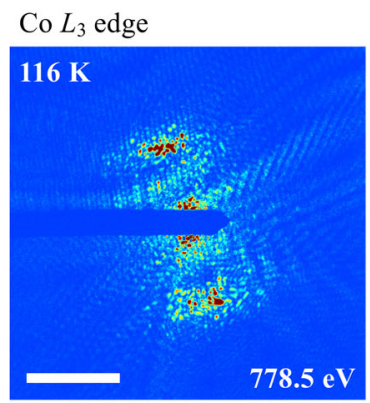

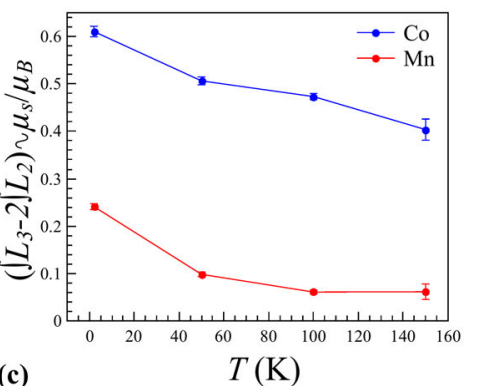

(c)

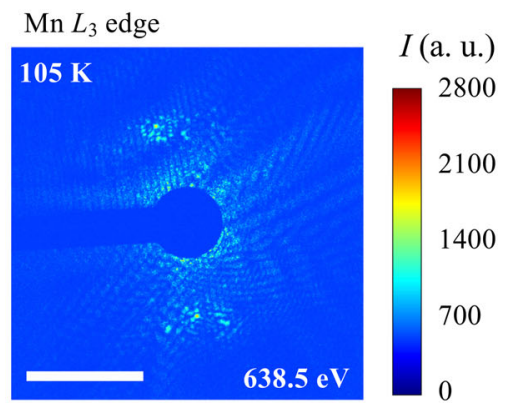

Fig. 3 X-ray spectroscopy and scattering from $\mathrm{Co}_{7} \mathrm{Zn}_{7} \mathbf{M n}_{6}$. XAS and XMCD spectra of $\mathrm{Co}_{7} \mathrm{Zn}_{7} \mathrm{Mn}_{6}$ measured near (a) Co and (b) Mn $L_{2,3}$ edges at $H=50 \mathrm{kOe}$. c Temperature dependence of XMCD signals $\left[L_{3}-2\left[L_{2}\right.\right.$ of Co and Mn integrated across corresponding edges. Error bars indicate the statistical error. d Schematic geometry of the REXS experiment. e Zero-field REXS patterns measured at Co $(E=776.5 \mathrm{eV}$ ) (left panel) and $\mathrm{Mn}(E=638.5 \mathrm{eV})$ (right panel) edges at various temperatures. The white scale bar corresponds to reciprocal space distance of $0.01 \AA^{-1}$. The central part of the scattering patterns is shadowed by the beamstop.

The $T$-dependent X-ray magnetic circular dichroism (XMCD) was measured on field warming at $50 \mathrm{kOe}$ in the field-polarized phase. Figure $3 \mathrm{a}, \mathrm{b}$ shows that at $2 \mathrm{~K}$ we observe a relatively strong absorption of the X-ray absorption spectroscopy (XAS)-normalized XMCD of $\approx 20 \%$ at the $C$ o $L_{3}$ edge, and $\approx 10 \%$ at the $M n L_{3}$ edge. Multiplet features observed in the Mn XMCD spectra (Fig. 3b) suggest that the $\mathrm{Mn} 2 p-3 d$ transition results from localization of the $\mathrm{Mn} 3 d$ electrons. Most features in the Mn spectrum are similar to those calculated for a $d^{5}$ ionic state, except the small pre-edge peak at $E=637.5 \mathrm{eV}$, which may result from a crystal field effect $^{54,55}$. Meanwhile, the Co $L_{3}$ and $L_{2}$ XMCD peak shapes are similar to the broad spectrum of metallic $\mathrm{Co}^{56}$. To evaluate a possible contribution of an oxidized surface layer, reference XAS and XMCD spectra were measured for the isostructural roomtemperature chiral magnet $\mathrm{Co}_{9} \mathrm{Zn}_{9} \mathrm{Mn}_{2}$ (see Supplementary note 4). The strong FM response observed at both $\mathrm{Co}$ and $\mathrm{Mn}$ edges in this compound indicates the dominant contribution of the target CoZnMn material to the XMCD signal, compared with any contribution from the oxidized surface.

To estimate the orbital to spin moment ratio we follow the approach given in ref. ${ }^{56}$ and find $\mu_{\mathrm{l}} / \mu_{\mathrm{s}}=0.260 \pm 0.012$ (Co) and $\mu_{\mathrm{l}} /$ $\mu_{\mathrm{s}}=0.0107 \pm 0.0045(\mathrm{Mn})$. For the latter, we assume a correction factor of 1.47 for the spin moment ${ }^{57}$. The same sign of measured high-field XMCD signal suggests a FM orientation of the Co and Mn sub-lattices relative to each other at high $H$. Element-selective magnetization loops are shown in Supplementary Fig. 6. Previously reported density functional theory calculations for $\mathrm{Co}_{7} \mathrm{Zn}_{7} \mathrm{Mn}_{6}$ anticipated $\mathrm{Mn}$ spin magnetic moments of $3.2 \mu_{\mathrm{B}}$ for the $12 d$ site, and Co moments of $1.3 \mu_{B}$ for the $8 c$ site ${ }^{23}$. However, the small magnitude of the XMCD signal from $\mathrm{Mn}$, as well as the neutron diffraction measurements in ref. ${ }^{23}$ suggest that $\mathrm{Mn}$ moments are not fully polarized even in a high external magnetic field. This scenario is consistent with the $\mu \mathrm{SR}$ data whereby there is a weak low $H$ dependence of $\lambda_{\mathrm{L}}$ just above $T_{\mathrm{g}}$. Energy-integrated XMCD signals at both $\mathrm{Co}$ and $\mathrm{Mn}$ edges are proportional to the corresponding spin moments $\int L_{3} d E-2 \int L_{2} d E \sim \mu_{\mathrm{s}} / \mu_{\mathrm{B}}$ and Fig. $3 \mathrm{C}$ shows that each becomes significantly reduced at higher $T$.
Notably, a large increment of Mn spin moment is found below $T_{\mathrm{g}}$ when fluctuations are suppressed.

Next, we turn to transmission resonant elastic X-ray scattering (REXS) to identify the magnetic elements responsible for the nanometric helical and skyrmion phases in $\mathrm{Co}_{7} \mathrm{Zn}_{7} \mathrm{Mn}_{6}{ }^{15,23}$. Figure $3 \mathrm{~d}$ shows a sketch of the experimental geometry, and Fig. $3 e$ shows REXS patterns obtained at either the Co or Mn edges. Each pattern shows magnetic X-ray scattering peaks due to helical order with $Q=0.0112 \AA^{-1}$ in a similar $T$ range above $100 \mathrm{~K}$. The length of the propagation vector corresponds to a real-space periodicity of $56 \mathrm{~nm}$, which is different from that in bulk samples, but consistent with Lorentz microscopy data reported for the thin plates $^{15}$. Below $90 \mathrm{~K}$ a magnetic REXS signal could not be distinguished from the remaining background charge scattering and charge-magnetic scattering interference.

Notably, the data in Fig. 3e show that the scattering from the helical order arises due to both magnetic elements, and is clearly at the $M n L_{3}$ absorption edge $(E=638.5 \mathrm{eV})$. Since only a weak $\mathrm{XMCD}$ signal is found for $\mathrm{Mn}$ at higher $T$, both the optical theorem and Kramers-Kronig relations imply that both imaginary and real parts of the refractive index have small magnetic contributions ${ }^{58}$. Indeed, when shown in the same intensity scale in Figure $3 e$, the element-selective REXS patterns clearly demonstrate the dominant contribution of $\mathrm{Co}$ ions in forming the helical order. The $2 p$ core hole absorption for the L-edge XMCD and REXS measurements occur over fs time scale thus not being sensitive to the $\mu \mathrm{s}$ fluctuation dynamics observed by the $\mu \mathrm{SR}$.

Therefore, from the combination of XMCD and REXS data from $\mathrm{Co}_{7} \mathrm{Zn}_{7} \mathrm{Mn}_{6}$, we confirm the dominant role of $\mathrm{Co}$ in forming helical, and thus skyrmion structures in finite $H$. This implicates the majority of $\mathrm{Mn}$ moments instead contribute to the static shortrange spin correlations observed by MDNS, and the slow $\mu \mathrm{s}$ magnetic fluctuations observed by $\mu \mathrm{SR}$. The X-ray data nonetheless imply a minority of $\mathrm{Mn}$ do in fact modulate helically, presumably this is due to a small Mn occupancy at the $8 \mathrm{c}$ site and thus a FM coupling with Co. These results are consistent with previous studies of $\mathrm{Co}_{8} \mathrm{Zn}_{8} \mathrm{Mn}_{4}{ }^{59}$, and ab-initio calculations ${ }^{23}$. We 

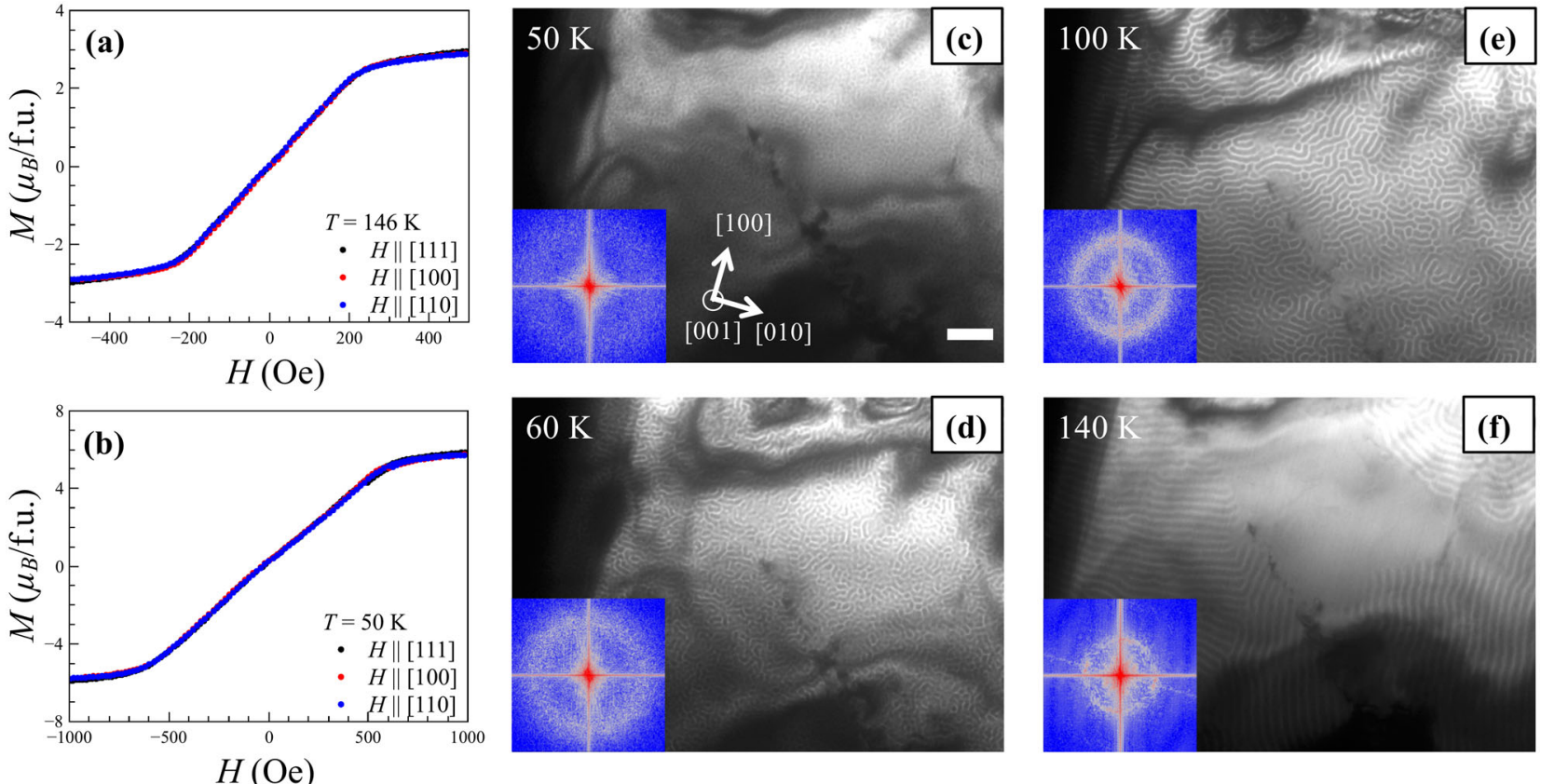

Fig. 4 Magnetization curves and Lorentz microscopy. Magnetization curves of bulk $\mathrm{Co}_{7} \mathrm{Zn}_{7} \mathrm{Mn}_{6}$ measured for principal crystallographic axes at (a) $146 \mathrm{~K}$ and (b) $50 \mathrm{~K}$. Evolution of the magnetic textures in thin plate $\mathrm{Co}_{7} \mathrm{Zn}_{7} \mathrm{Mn}_{6}$ measured on zero-field warming at (c) $50 \mathrm{~K}$, d $60 \mathrm{~K}$, e $100 \mathrm{~K}$ and (f) $140 \mathrm{~K}$. The white scale bar corresponds to $500 \mathrm{~nm}$. The insets show corresponding fast Fourier transform patterns. Details of the LTEM measurement protocol are given in "Methods".

thus conclude that the LTSk phase in $\mathrm{Co}_{7} \mathrm{Zn}_{7} \mathrm{Mn}_{6}$ is composed principally of modulating Co moments at the $8 c$ site, while the LTSk phase stability relies on a coupling between Co and fluctuating $\mathrm{Mn}$ moments that mainly occupy the $12 d$ site, with this coupling deduced to be FM from the XMCD data.

\section{Unimportance of low-temperature cubic anisotropy}

Next we consider the importance of low $T$ cubic anisotropies, which recent theory shows can stabilize a low $T$ equilibrium skyrmion phase chiral magnet in $\mathrm{Cu}_{2} \mathrm{OSeO}_{3}{ }^{60-62}$. Figure $4 a, b$ shows magnetization curves measured on bulk single crystal $\mathrm{Co}_{7} \mathrm{Zn}_{7} \mathrm{Mn}_{6}$ for $H\|[111], H\|[110], H \|[100]$. Details of the measurement are given in the "Methods". From these data, no clear sign of magnetic anisotropy is seen at either $T=146 \mathrm{~K}$ and $T=50 \mathrm{~K}$, these being Ts where the A-phase and LTSk phases are stable, respectively. This contrasts with similar data from $\mathrm{Cu}_{2} \mathrm{OSeO}_{3}$, where the importance of low $T$ cubic and exchange anisotropies shows up firstly as a clear anisotropy of the $\mathrm{H}$-dependent magnetization between the principal crystal directions ${ }^{61}$, and secondly by the observation that the LTSk phase is energetically favoured over the helical and conical states only for $H \|[100]^{60,62}$.

As further evidence for the weak influence of low $T$ cubic anisotropy in $\mathrm{Co}_{7} \mathrm{Zn}_{7} \mathrm{Mn}_{6}$, we turn to real-space LTEM images of the magnetic textures in a $\sim 150 \mathrm{~nm}$ thin-plate sample-see "Methods" for further experimental details. Figur 4c-f shows LTEM images obtained in $H=0$ and on warming from $T=50 \mathrm{~K}$ to $140 \mathrm{~K}$. At $T=50 \mathrm{~K}$, just above $T_{\mathrm{g}}$, Fig. 4(c) shows an image of a highlydisordered helical state. Such a state hosting a large number of topological defects can provide a fertile soil for the nucleation of the LTSk phase in finite $H^{63}$. Similar disordered magnetic textures were observed after ZFC (see Supplementary note 6). On warming, this ZF texture evolves into multiple worm-like helical domains (Fig. 4d, e), before finally forming well-defined helical stripes propagating along the cubic axes at $140 \mathrm{~K}$ (Fig. 4f). This $T$ evolution of the magnetic contrast suggests that near $T_{\mathrm{g}}$, the helical texture has multiple local energy minima, likely introduced by frustration-induced magnetic disorder, while nearer to $T_{\mathrm{c}}$ thermal energy is sufficient to drive the re-orientation of wellordered helices along the cubic axes as preferred by magnetic anisotropy.

According to the theory given in ref. ${ }^{60}$, the LTSk phase stabilizes when the anisotropy energy density $K$ exceeds the critical value $K_{\mathrm{c}}=0.07 \mu_{0} H_{\mathrm{c} 2} M_{\mathrm{s}}$, where $H_{\mathrm{c} 2}$ is the critical field of the conical to a field-polarized phase transition, and $M_{\mathrm{s}}$ is the saturation magnetization, resulting in $K_{\mathrm{c}} \approx 2.7 \mathrm{~kJ} \mathrm{~m}^{-3}$ for $\mathrm{Co}_{7} Z_{7} \mathrm{Mn}_{6}$. Recently, the value of $K \approx 0.7 \mathrm{~kJ} \mathrm{~m}^{-3}$ was determined experimentally for $\mathrm{Co}_{7} \mathrm{Zn}_{7} \mathrm{Mn}_{6}$ by means of FM resonance spectroscopy ${ }^{64}$, which is well below the theoretical threshold of the anisotropy needed to stabilize the LTSk phase.

Taken together, the magnetization data from a bulk sample, and the LTEM data from a thin plate sample, each imply a weak influence of cubic (and exchange) anisotropies at low $T$ in $\mathrm{Co}_{7} \mathrm{Zn}_{7} \mathrm{Mn}_{6}$. In turn, this suggests such anisotropies as less important for the LTSk phase stability than the geometric magnetic frustration effects established by our experiments.

In summary, our quantum beam studies of $\mathrm{Co}_{7} \mathrm{Zn}_{7} \mathrm{Mn}_{6}$ provide convincing evidence that geometric frustration-induced magnetic fluctuations are a key ingredient for stabilizing the LTSk in this system. The crucial role of magnetic fluctuations is suggested from muon spin relaxation experiments, which reveal a pronounced increase in magnetic relaxation rate over the thermal range of LTSk phase stability. From the analysis of both muon and magnetic neutron diffuse scattering data, we connect the physical origin of the magnetic fluctuations to the geometric frustration of the $\mathrm{Mn}$ moments on the hyper-kagomé coordinated $12 d$ crystallographic site. This picture is corroborated by element-selective synchrotron X-ray experiments that reveal a FM coupling between the said Mn moments, and Co moments that occupy the different $8 c$ crystallographic site and modulate to form the helical and skyrmion phases. Finally, from both bulk magnetization and electron microscopy experiments, scant evidence is obtained for a significant role of cubic or exchange anisotropies on the magnetic properties in the thermal range of LTSk phase stability.

Overall, our data do not support an understanding of LTSk phase stability in terms of the leading theories that require either 
thermal fluctuations $s^{9,17-21}$ or low $T$ cubic anisotropies ${ }^{60-62}$. Instead, our experiments advocate the existence of a frustrationassisted skyrmion phase stability mechanism in $\mathrm{Co}_{7} \mathrm{Zn}_{7} \mathrm{Mn}_{6}$ that is based on frustration-induced magnetic fluctuations, and which calls for a detailed theoretical analysis. The elaboration of a such a stability mechanism could find general relevance in the ongoing research into topological magnetic textures amongst the multifarious frustrated magnets.

\section{METHODS}

\section{Bulk sample preparation}

Bulk single crystals of $\mathrm{Co}_{7} \mathrm{Zn}_{7} \mathrm{Mn}_{6}$ and $\mathrm{Co}_{8} \mathrm{Zn}_{8} \mathrm{Mn}_{4}$ were grown by the Bridgman method as described in refs. ${ }^{13,15}$ and studied in previous SANS experiments. The image of the crystal structure shown in Fig. 1a was depicted using VESTA software ${ }^{65}$.

A bulk polycrystalline $\mathrm{Co}_{7} \mathrm{Zn}_{7} \mathrm{Mn}_{6}$ specimen for X-ray spectroscopy was obtained from an ingot. Thin sample preparation for the REXS experiment is described in the soft $\mathrm{X}$-ray experiment section.

$\mathrm{Co}_{7} \mathrm{Zn}_{7} \mathrm{Mn}_{6}$ powder was prepared from the polycrystalline ingots for the MDNS experiment.

\section{$\boldsymbol{\mu S R}$ experiment}

In the present $\mu \mathrm{SR}$ experiments we have used the same single crystals of $\mathrm{Co}_{7} \mathrm{Zn}_{7} \mathrm{Mn}_{6}$ and $\mathrm{Co}_{8} \mathrm{Zn}_{8} \mathrm{Mn}_{4}$ produced by the Bridgman method as studied in the previous SANS studies refs. ${ }^{13,15}$. The spectra were measured using the GPS muon instrument at $S \mu S$ (Paul Scherrer Institute, Villigen, Switzerland) ${ }^{66}$. The samples were placed into $\mathrm{Cu}$ envelopes and attached to a sample holder. The crystals were oriented with [001] axis parallel to the incoming muon beam and LF. In a ZF experiment, spin-polarized muons $\left(\mu^{+}\right)$are implanted in a magnetic specimen at normal incidence. The spins of the implanted muons precess about the local magnetic field at the $\mu^{+}$site, thus, being sensitive to the local field distribution. Statically disordered and fluctuating local magnetic moments depolarize the muons. In a LF experiment, the external magnetic field is applied in the direction of the initial polarization of the $\mu^{+}$. Muons implanted into the sample decay into positrons, which are emitted preferentially in the direction of the $\mu^{+}$ spin. In the actual ZF and LF experiments, the emitted positrons are detected along with directions both parallel and anti-parallel (forward and backward directions) to the initial muon spin direction. The observed evolution of the asymmetry $A(t)$ (difference between the number of positrons in the forward and backward directions) depends on both static and dynamical components of the local spin arrangement in the material. The maximum asymmetry and efficiencies of the forward and backward positron detectors were determined from standard calibration measurements made in the paramagnetic state at $T=250 \mathrm{~K}$ and $T=320 \mathrm{~K}$ for $\mathrm{Co}_{7} \mathrm{Zn}_{7} \mathrm{Mn}_{6}$ and $\mathrm{Co}_{8} \mathrm{Zn}_{8} \mathrm{Mn}_{4}$, respectively, with a small applied transverse magnetic field $H=20$ Oe. The $\mu$ SR spectra were analyzed using the Musrfit software $^{67}$.

\section{Diffuse neutron scattering}

MDNS experiments over a $Q$ range between 0.5 and $3.8 \AA^{-1}$ were carried out at D7 instrument at the Institut Laue-Langevin (Grenoble, France) ${ }^{50}$ using $x y z$ polarization analysis to isolate the magnetic component of the scattering. A powdered $\mathrm{Co}_{7} \mathrm{Zn}_{7} \mathrm{Mn}_{6}$ sample was loaded into double-wall aluminium container and loaded into an Orange-type He cryostat. For the experiment, an incoming neutron wavelength of $3.1 \AA$ was used.

\section{Mean-field model}

The MF calculation for paramagnetic scattering is similar to that of ref. ${ }^{68}$ following the general formalism of ref. ${ }^{69}$, and constitutes a phenomenological Heisenberg model of isotropically interacting local magnetic $\mathrm{Mn}$ moments at the $12 d$ positions $\left(\mathbf{R}+\mathbf{r}^{a}\right)$ in the average crystal structure ${ }^{23,24,70}$. Here $\mathbf{R}$ is a primitive lattice translation vector, $\mathbf{r}$ is a vector separation between spins, and $a \in\{1,12\}$ are the site indices. Refinements were parameterized by a set of exchange constants, $J_{a b}\left(\mathbf{R}, \mathbf{R}^{\prime}\right)$, while the eigenvectors and eigenvalues of the $12 \times 12$ reciprocal space interaction matrix $J_{a b}(\mathbf{q})=\sum_{\mathbf{R}} J_{a b}(\mathbf{R}, 0) \exp (i \mathbf{q} \cdot \mathbf{R})$ were used to evaluate the MF expression for the diffuse scattering intensity ${ }^{68}$. To perform the spherical average needed for the powder spectrum, the binning method of ref. ${ }^{71}$ was used. Optimal values of the exchange constants were obtained by direct fitting to the experimental data, using a simulated annealing algorithm ${ }^{72}$.

\section{RMC analysis}

The MDNS data were fitted via RMC approach ${ }^{68}$ and the distancedependent radial spin-pair correlation function is shown in Fig. S3. Note that, due to the limited amount of information that can be extracted from the MDNS profile, for both the RMC analysis and MF calculation, we used a simplified model that assumes the MDNS arises from only a $12 d$ site that is fully occupied by magnetic $\mathrm{Mn}$ atoms. While this does not represent the true situation, we expect that the near half-filling of the $12 d$ site with $\mathrm{Mn}$ is sufficient to induce frustration on the atomic lengthscale that is sufficient to affect the nanoscale chiral magnetism.

\section{Resonant soft X-ray scattering}

The REXS in transmission geometry was measured using SEXTANTS beamline (Sychrotron SOLEIL, France) on $\mathrm{Co}_{7} \mathrm{Zn}_{7} \mathrm{Mn}_{6}$ lamella fabricated by focused ion beam (FIB) lithography using Hitachi NB5000 setup (Fig. S5). Commercial silicon nitride membranes from Silson Ltd (Southam, UK) were processed for soft X-ray experiments. The front side of each membrane was coated with $\approx 4 \mu \mathrm{m}$-thick layer of gold to absorb the incoming beam. An $\sim 100 \mathrm{~nm}$-thick lamella of $\mathrm{Co}_{7} \mathrm{Zn}_{7} \mathrm{Mn}_{6}$ alloy with a surface containing (001) plane was cut from the bulk single crystal. An aperture with a diameter of $3.5 \mu \mathrm{m}$ and asymmetric shape was drilled in the gold coating of the membrane to provide the transmission through the sample (Fig. S5a). The lamella was fixed to the membrane by using single tungsten contact to reduce the influence of the tensile strain ${ }^{73}$ (Fig. S5b). The small size and asymmetric shape of the aperture were chosen for the real-space reconstructions of magnetization textures by means of phase-retrieval algorithm ${ }^{59,74,75}$. Unfortunately, poor signal-to-noise ratio due to the background charge and charge-magnetic interference scattering did not allow real-space images to be obtained.

The RESOXS chamber at SEXTANTS beamline is equipped with a highvacuum chamber with a background pressure of $10^{-9} \mathrm{mbar}^{53}$. The scattered intensity was collected by an in-vacuum charge-coupled device area $x$-ray detector of $2048 \times 2048$ pixels (Princeton Instruments). An aluminium beamstop was introduced to protect the central part of the detector for the REXS experiment. The magnetic field provided by the electromagnet was applied perpendicular to the lamella surface and parallel to the incident circularly polarized X-ray beam (Fig. S6a). A He-flowtype cryostat was used to control the sample temperature.

To subtract the background arising from the charge scattering we have measured the scattering patterns with magnetic field of $H=1.6 \mathrm{kOe}$ applied perpendicular to the sample plane corresponding to the induced FM state of the system. The sample position was corrected after each temperature change by using the transmission signal detected by a photodiode.

\section{X-ray magnetic circular dichroism}

The XMCD experiment was carried out at XTreme beamline at Swiss light source (Villigen, Switzerland) equipped with a vector-field cryomagnet. The beamline is dedicated to polarization-dependent soft XAS at a high magnetic field and low temperature ${ }^{52}$.

Polarization-dependent X-ray absorption spectra were measured with energy resolution of $0.1 \mathrm{eV}$ using the surface-sensitive total electron yield method near $C o$ and $M n L_{2,3}$ edges with right and left circularly polarized Xrays. To maximize the XMCD signal, the sample was cooled down to a temperature of $2 \mathrm{~K}$ under a magnetic field of $50 \mathrm{kOe}$ applied along the $\mathrm{X}$ ray beam and [110] crystallographic axis of the sample. Prior to the experiment, the sample was polished to minimize the effect of the surface oxidation. All XAS spectra were normalized to their corresponding edge jumps between the pre- $L_{3}$ and post- $L_{2}$ regions. All XMCD spectra were normalized to their corresponding XAS.

\section{Magnetization measurements}

DC magnetization measurements for a $\mathrm{Co}_{7} \mathrm{Zn}_{7} \mathrm{Mn}_{6}$ single crystal were performed using the vibrating sample magnetometer mode of a superconducting quantum interference device magnetometer (SQUID MPMS3, Quantum Design). Magnetic field values in the $M(H)$ curves (Fig. $4 a$, b) for $H \|[111]$ are calibrated by the demagnetization factor of $0.82 \mathrm{H}$. Note that the difference in saturation field values in Figs. $1 b$ and $3 a, b$ is because of 
the difference between demagnetization factors for the samples used in the $M(H)$ and $\mu \mathrm{SR}$ measurements.

\section{Lorentz microscopy}

LTEM measurements were performed on a FIB-made 150 nm-thick (001)oriented lamella with a transmission electron microscope (JEM-2100F) at an acceleration voltage of $200 \mathrm{kV}$. Defocus distances for LTEM images were set at $-288 \mu \mathrm{m}$ (Fig. 4c) and $-480 \mu \mathrm{m}$ (Fig. 4d-f). A liquid-helium cooling double-tilt holder was used to obtain LTEM images at low temperature. Figure $4 \mathrm{c}-\mathrm{f}$ were measured on zero-field warming after saturating $\mathrm{Co}_{7} \mathrm{Zn}_{7} \mathrm{Mn}_{6}$ sample by the magnetic field of $H=2.85 \mathrm{kOe}$ applied perpendicular to the sample plane at $50 \mathrm{~K}$. Therefore, a certain fraction of metastable skyrmions embedded in the helical phase is observed up to $T \sim 120 \mathrm{~K}$.

\section{DATA AVAILABILITY}

All experimental data presented in the figures that support the findings of this study are available at the https://doi.org/10.5281/zenodo.4627948. MDNS raw data is also available from the ILL repository ${ }^{49}$.

Received: 2 November 2020; Accepted: 31 March 2021; Published online: 23 April 2021

\section{REFERENCES}

1. Rößler, U. K., Bogdanov, A. \& Pfleiderer, C. Spontaneous skyrmion ground states in magnetic metals. Nature 442, 797 (2006).

2. Nagaosa, N. \& Tokura, Y. Topological properties and dynamics of magnetic skyrmions. Nat. Nanotechnol. 8, 899 (2013).

3. Kanazawa, N., Seki, S. \& Tokura, Y. Noncentrosymmetric magnets hosting magnetic skyrmions. Adv. Mater. 29, 1603227 (2017).

4. Jonietz, F. et al. Spin transfer torques in MnSi at ultralow current densities. Science 330, 1648-1651 (2010)

5. Schwarze, T. et al. Universal helimagnon and skyrmion excitations in metallic semiconducting and insulating chiral magnets. Nat. Mater. 14, 478 (2015)

6. Mochizuki, M. et al. Thermally driven ratchet motion of a skyrmion microcrystal and topological magnon hall effect. Nat. Mater. 13, 241-246 (2014).

7. Fert, A., Reyren, N. \& Cros, V. Magnetic skyrmions: advances in physics and potential applications. Nat. Rev. Mater. 2, 17031 (2017).

8. Huang, Y., Kang, W., Zhang, X., Zhou, Y. \& Zhao, W. Magnetic skyrmion-based synaptic devices. Nanotechnology 28, $08 \mathrm{LT02}$ (2017).

9. Mühlbauer, S. et al. Skyrmion lattice in a chiral magnet. Science 323, 915-919 (2009)

10. $\mathrm{Yu}, \mathrm{X}$. Z. et al. Near room-temperature formation of a skyrmion crystal in thinfilms of the helimagnet FeGe. Nat. Mater. 10, 106-109 (2011).

11. Seki, S., Yu, X. Z., Ishiwata, S. \& Tokura, Y. Observation of skyrmions in a multiferroic material. Science 336, 198-201 (2012).

12. Tokunaga, $Y$. et al. A new class of chiral materials hosting magnetic skyrmions beyond room temperature. Nat. Commun. 6 (2015).

13. Karube, $\mathrm{K}$. et al. Robust metastable skyrmions and their triangular-square lattice structural transition in a high-temperature chiral magnet. Nat. Mater. 15, 1237 (2016).

14. Karube, K. et al. Skyrmion formation in a bulk chiral magnet at zero magnetic field and above room temperature. Phys. Rev. Mater. 1, 074405 (2017).

15. Karube, K. et al. Disordered skyrmion phase stabilized by magnetic frustration in a chiral magnet. Sci. Adv. 4, eaar7043 (2018).

16. Karube, $\mathrm{K}$. et al. Metastable skyrmion lattices governed by magnetic disorder and anisotropy in $\beta$-Mn-type chiral magnets. Phys. Rev. B 102, 064408 (2020).

17. Rößler, U. K., Leonov, A. A. \& Bogdanov, A. N. Chiral skyrmionic matter in noncentrosymmetric magnets. J. Phys. Confer. Ser. 303, 012105 (2011).

18. Buhrandt, S. \& Fritz, L. Skyrmion lattice phase in three-dimensional chiral magnets from monte carlo simulations. Phys. Rev. B 88, 195137 (2013).

19. Keesman, R. et al. Degeneracies and fluctuations of Néel skyrmions in confined geometries. Phys. Rev. B 92, 134405 (2015).

20. Laliena, V., Albalate, G. \& Campo, J. Stability of the skyrmion lattice near the critical temperature in cubic helimagnets. Phys. Rev. B 98, 224407 (2018).

21. Kruchkov, A. et al. Direct electric field control of the skyrmion phase in a magnetoelectric insulator. Sci. Rep. 8, 1-7 (2018).

22. Roldán-Molina, A., Santander, M., Nunez, A. \& Fernández-Rossier, J. Quantum fluctuations stabilize skyrmion textures. Phys. Rev. B 92, 245436 (2015).
23. Bocarsly, J. D., Heikes, C., Brown, C. M., Wilson, S. D. \& Seshadri, R. Deciphering structural and magnetic disorder in the chiral skyrmion host materials $\mathrm{Co}_{x} \mathrm{Zn}_{y} \mathrm{Mn}_{z}$ $(x+y+z=20)$. Phys. Rev. Mater. 3, 014402 (2019).

24. Nakajima, T. et al. Correlation between site occupancies and spin-glass transition in skyrmion host $\mathrm{Co}_{10-x / 2} \mathrm{Zn}_{10-x / 2} \mathrm{Mn}_{x}$. Phys. Rev. B 100, 064407 (2019).

25. Nakamura, H., Yoshimoto, K., Shiga, M., Nishi, M. \& Kakurai, K. Strong antiferromagnetic spin fluctuations and the quantum spin-liquid state in geometrically frustrated- $\beta-\mathrm{Mn}$, and the transition to a spin-glass state caused by nonmagnetic impurity. J. Phys. Condens. Matter 9, 4701 (1997).

26. Stewart, J., Rainford, B., Eccleston, R. \& Cywinski, R. Non-fermi-liquid behavior of electron-spin fluctuations in an elemental paramagnet. Phys. Rev. Lett. 89, 186403 (2002).

27. Paddison, J. A. et al. Emergent frustration in Co-doped $\beta$-Mn. Phys. Rev. Lett. 110, 267207 (2013).

28. Mekata, M. et al. Spin freezing in geometrically frustrated $\beta$-Mn probed by muon spin relaxation. Hyperfine Interact. 120, 639-643 (1999).

29. Yaouanc, A. \& Dalmas de Réotier, P. Muon Spin Rotation, Relaxation, and Resonance: Applications to Condensed Matter. International Series of Monographs on Physics (Oxford Univ. Press, Oxford, 2011).

30. Johnston, D. Stretched exponential relaxation arising from a continuous sum of exponential decays. Phys. Rev. B 74, 184430 (2006).

31. Uemura, Y., Yamazaki, T., Harshman, D., Senba, M. \& Ansaldo, E. Muon-spin relaxation in AuFe and CuMn spin glasses. Phys. Rev. B 31, 546 (1985).

32. Liu, L. $\mu$ SR Study of B2O Magnetic Systems: MnSi, $\mathrm{Mn}_{0.9} \mathrm{Fe}_{0.1} \mathrm{Si}_{\text {and }} \mathrm{Cu}_{2} \mathrm{OSeO}_{3}$. Ph.D. thesis (Columbia University, New York, 2016).

33. Martin, N. et al. Magnetic ground state and spin fluctuations in MnGe chiral magnet as studied by muon spin rotation. Phys. Rev. B 93, 174405 (2016).

34. Lancaster, T. et al. Transverse field muon-spin rotation measurement of the topological anomaly in a thin film of MnSi. Phys. Rev. B 93, 140412 (2016).

35. Franke, K. J. et al. Magnetic phases of skyrmion-hosting $\mathrm{GaV}_{4} \mathrm{~S}_{8-y} \mathrm{Se}_{y}(\mathrm{y}=0,2,4$, 8) probed with muon spectroscopy. Phys. Rev. B 98, 054428 (2018).

36. Hicken, $\mathrm{T}$. et al. Magnetism and Néel skyrmion dynamics in $\mathrm{GaV}_{4} \mathrm{~S}_{8-y} \mathrm{Se}_{y}$. Phys. Rev. Res. 2, 032001 (2020)

37. Mirebeau, I. et al. Depolarization in reentrant spin glasses: a comparison between neutron and muon probes. Hyperfine Interact. 104, 343-348 (1997).

38. Stewart, J. \& Cywinski, R. $\mu$ SR evidence for the spin-liquid-to-spin-glass transition in $\beta-\mathrm{Mn}_{1-x} \mathrm{Al}_{x}$. Phys. Rev. B 59, 4305 (1999).

39. Fudamoto, $\mathrm{Y}$. et al. Static spin freezing in $\mathrm{NaV}_{2} \mathrm{O}_{5}$ detected by muon spin relaxation. Phys. Rev. Lett. 83, 3301 (1999).

40. Ryan, D., Cadogan, J. \& Van Lierop, J. Muon spin resonance study of transverse spin freezing in $a-\mathrm{Fe}_{x} \mathrm{Zr}_{100-x}$. Phys. Rev. B 61, 6816 (2000).

41. Rotaru, $\mathrm{G}$. et al. Spin-glass state and long-range magnetic order in $\mathrm{Pb}\left(\mathrm{Fe}_{1 / 2} \mathrm{Nb}_{1 / 2}\right)$ $\mathrm{O}_{3}$ seen via neutron scattering and muon spin rotation. Phys. Rev. B 79, 184430 (2009).

42. Kalvius, $\mathrm{G}$. et al. Magnetism of frustrated A-site spinels ( $\mathrm{Mn}, \mathrm{Fe}, \mathrm{Co}) \mathrm{Al}_{2} \mathrm{O}_{4}$. Phys. B: Condens. Matter 404, 660-662 (2009).

43. Sannigrahi, J. et al. Magnetic states of Ni-Mn-Sn based shape memory alloy: a combined muon spin relaxation and neutron diffraction study. Phys. Rev. B 99, 224401 (2019).

44. Rainford, B., Stewart, J., Leavey, C. \& Hillier, A. $\mu$ SR study of the onset of magnetic order in $\beta$-Mn-Ru alloys. J. Magn. Magn. Mater. 310, 1314-1315 (2007).

45. Leavey, C., Stewart, J., Rainford, B. \& Hillier, A. Magnetic ground states and spin dynamics of $\beta-\mathrm{Mn}_{1-x}$ Rux alloys. J. Phys.: Condens. Matter 19, 145288 (2007).

46. Hafner, J. \& Hobbs, D. Understanding the complex metallic element $\mathrm{Mn}$. II. geometric frustration in $\beta-\mathrm{Mn}$, phase stability, and phase transitions. Phys. Rev. $B$ 68, 014408 (2003).

47. Zhang, $\mathrm{R}$. et al. Spin liquids and spin glasses in Mn-based alloys with the cubic A13 ( $\beta M n)$ structure. J. Magn. Magn. Mater. 501, 166429 (2020).

48. Yamauchi, $\mathrm{H}$. et al. High-temperature short-range order in $\mathrm{Mn}_{3} \mathrm{RhSi}$. Commun Mater. 1, 1-6 (2020).

49. White, J. S., Karube, K., Rønnow, H. M. \& Wildes, A. From chiral helimagnet to frustrated spin liquid by tuning the $\mathrm{Mn}$ concentration in $\mathrm{Co}_{x} \mathrm{Zn}_{y} \mathrm{Mn}_{z}$ alloys. Institut Laue-Langevin (ILL) https://doi.org/10.5291/ILL-DATA.5-32-855 (2018).

50. Stewart, J. et al. Disordered materials studied using neutron polarization analysis on the multi-detector spectrometer, D7. J. Appl. Crystallogr. 42, 69-84 (2009).

51. Bramwell, S. T. Neutron Scattering and Highly Frustrated Magnetism, 45-78 (Springer, 2011).

52. Piamonteze, $C$. et al. X-Treme beamline at SLS: X-ray magnetic circular and linear dichroism at high field and low temperature. J. Synchrotron Radiat. 19, 661-674 (2012).

53. Jaouen, $N$. et al. An apparatus for temperature-dependent soft x-ray resonant magnetic scattering. J. Synchrotron Radiat. 11, 353-357 (2004).

54. van der Laan, G. \& Thole, B. Strong magnetic x-ray dichroism in $2 p$ absorption spectra of 3d transition-metal ions. Phys. Rev. B 43, 13401 (1991). 
55. Holm, A. P. et al. XMCD characterization of the ferromagnetic state of $\mathrm{Yb}_{14} \mathrm{MnSb}_{11}$. J. Am. Chem. Soc. 124, 9894-9898 (2002).

56. Thole, B., Carra, P., Sette, F. \& van der Laan, G. X-ray circular dichroism as a probe of orbital magnetization. Phys. Rev. Lett. 68, 1943 (1992).

57. Teramura, Y., Tanaka, A. \& Jo, T. Effect of coulomb interaction on the x-ray magnetic circular dichroism spin sum rule in $3 \mathrm{~d}$ transition elements. J. Phys. Soc. Jpn. 65, 1053-1055 (1996).

58. Fink, J., Schierle, E., Weschke, E. \& Geck, J. Resonant elastic soft x-ray scattering. Rep. Prog. Phys. 76, 056502 (2013).

59. Ukleev, V. et al. Element-specific soft x-ray spectroscopy, scattering, and imaging studies of the skyrmion-hosting compound $\mathrm{Co}_{8} \mathrm{Zn}_{8} \mathrm{Mn}_{4}$. Phys. Rev. B 99, 144408 (2019).

60. Chacon, A. et al. Observation of two independent skyrmion phases in a chiral magnetic material. Nat. Phys. 14, 936 (2018).

61. Halder, M. et al. Thermodynamic evidence of a second skyrmion lattice phase and tilted conical phase in $\mathrm{Cu}_{2} \mathrm{OSeO}_{3}$. Phys. Rev. B 98, 144429 (2018).

62. Bannenberg, L. J. et al. Multiple low-temperature skyrmionic states in a bulk chiral magnet. npj Quantum Mater. 4, 11 (2019).

63. Mirebeau, I. et al. Spin textures induced by quenched disorder in a reentrant spin glass: Vortices versus "frustrated" skyrmions. Phys. Rev. B 98, 014420 (2018).

64. Preißinger, M. et al. Vital role of anisotropy in cubic chiral skyrmion hosts. Preprint at http://arxiv.org/abs/2011.05967 (2020).

65. Momma, K. \& Izumi, F. VESTA 3 for three-dimensional visualization of crystal, volumetric and morphology data. J. Appl. Crystallogr. 44, 1272-1276 (2011).

66. Amato, A. et al. The new versatile general purpose surface-muon instrument (GPS) based on silicon photomultipliers for $\mu$ SR measurements on a continuouswave beam. Rev. Sci. Instrum. 88, 093301 (2017).

67. Suter, A. \& Wojek, B. Musrfit: a free platform-independent framework for $\mu$ SR data analysis. Phys. Procedia 30, 69-73 (2012).

68. Paddison, J. A., Stewart, J. R. \& Goodwin, A. L. SPINVERT: A program for refinement of paramagnetic diffuse scattering data. J. Phys.: Condens. Matter 25, 454220 (2013).

69. Enjalran, M. \& Gingras, M. J. Theory of paramagnetic scattering in highly frustrated magnets with long-range dipole-dipole interactions: the case of the $\mathrm{Tb}_{2} \mathrm{Ti}_{2} \mathrm{O}_{7}$ pyrochlore antiferromagnet. Phys. Rev. B 70, 174426 (2004).

70. Hori, T., Shiraish, H. \& Ishii, Y. Magnetic properties of $\beta$-MnCoZn alloys. J. Magn. Magn. Mater. 310, 1820-1822 (2007).

71. Reimers, J. N. Diffuse-magnetic-scattering calculations for frustrated antiferromagnets. Phys. Rev. B 46, 193 (1992).

72. Goffe, W. L., Ferrier, G. D. \& Rogers, J. Global optimization of statistical functions with simulated annealing. J. Econ. 60, 65-99 (1994).

73. Okamura, Y. et al. Emergence and magnetic-field variation of chiral-soliton lattice and skyrmion lattice in the strained helimagnet $\mathrm{Cu}_{2} \mathrm{OSeO}_{3}$. Phys. Rev. B 96, 174417 (2017)

74. Flewett, S. et al. Method for single-shot coherent diffractive imaging of magnetic domains. Phys. Rev. Lett. 108, 223902 (2012).

75. Ukleev, V. et al. Coherent resonant soft x-ray scattering study of magnetic textures in FeGe. Quantum Beam Sci. 2, 3 (2018).

\section{ACKNOWLEDGEMENTS}

V.U., P.M.D., L.Y., H.M.R. and J.S.W. acknowledge funding from the SNSF Sinergia CRSII5_171003 NanoSkyrmionics. This research was supported in part by JSPS Grantin-Aids for Scientific Research (Grant No. 20K15164) and JST CREST (Grant No. JPMJCR1874 and JPMJCR20T1). X-ray scattering experiments were carried out at beamline SEXTANTS at SOLEIL as a part of the Proposal No. 20181292. The research leading to this result has been supported by the project CALIPSOplus under the Grant Agreement 730872 from the EU Framework Programme for Research and Innovation HORIZON 2020. The authors wish to acknowledge P. Gargiani and M. Valvidares for providing element-selective magnetization measurements at BOREAS beamline (ALBA Synchrotron Light Source) and A. Zakharova for supporting experiment at Xtreme.

\section{AUTHOR CONTRIBUTIONS}

K.K. and A.K. synthesized the samples; V.U., C.N.W., J.S.W., H.L. performed and analyzed the muon measurements; K.K., L.M.-T., A.R.W., J.S.W. performed and analyzed the neutron scattering measurements; P.M.D. performed the mean-field calculations; K.K. performed magnetization measurements; D.M. performed electron microscopy experiments; D.M. and V.U. prepared the sample for X-ray scattering; V.U., Y.Yam., Y.Yok., L.Y., J.S.W., N.J. performed and analyzed REXS measurements; V.U. and C.P. performed and analyzed X-ray spectroscopy measurements; V.U. and J.S.W. wrote the manuscript with contributions from P.M.D., C.N.W., A.R.W., C.P., H.M.R., T.A., Y. Tokunaga, Y.Tag.; Y. Tokunaga, H.M.R., T.A., Y. Tokura, Y.Tag., J.S.W. jointly conceived the project.

\section{COMPETING INTERESTS}

The authors declare no competing interests.

\section{ADDITIONAL INFORMATION}

Supplementary information The online version contains supplementary material available at https://doi.org/10.1038/s41535-021-00342-5.

Correspondence and requests for materials should be addressed to V.U. or J.S.W.

Reprints and permission information is available at http://www.nature.com/reprints

Publisher's note Springer Nature remains neutral with regard to jurisdictional claims in published maps and institutional affiliations.

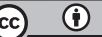

Open Access This article is licensed under a Creative Commons Attribution 4.0 International License, which permits use, sharing, adaptation, distribution and reproduction in any medium or format, as long as you give appropriate credit to the original author(s) and the source, provide a link to the Creative Commons license, and indicate if changes were made. The images or other third party material in this article are included in the article's Creative Commons license, unless indicated otherwise in a credit line to the material. If material is not included in the article's Creative Commons license and your intended use is not permitted by statutory regulation or exceeds the permitted use, you will need to obtain permission directly from the copyright holder. To view a copy of this license, visit http://creativecommons. org/licenses/by/4.0/.

(c) The Author(s) 2021 